\title{
Hybrid projective combination-combination synchronization in non-identical hyperchaotic systems using adaptive control
}

Received: 14 August 2019 / Accepted: 1 February 2020 / Published online: 5 March 2020

(C) The Author(s) 2020

\begin{abstract}
In this paper, we investigate a hybrid projective combination-combination synchronization scheme among four non-identical hyperchaotic systems via adaptive control method. Based on Lyapunov stability theory, the considered approach identifies the unknown parameters and determines the asymptotic stability globally. It is observed that various synchronization techniques, for instance, chaos control problem, combination synchronization, projective synchronization, etc. turn into particular cases of combination-combination synchronization. The proposed scheme is applicable to secure communication and information processing. Finally, numerical simulations are performed to demonstrate the effectivity and correctness of the considered technique by using MATLAB.
\end{abstract}

Mathematics Subject Classification $34 \mathrm{~K} 23 \cdot 34 \mathrm{~K} 35 \cdot 37 \mathrm{~B} 25 \cdot 37 \mathrm{~N} 35$

\section{Introduction}

Chaos is described as a nonlinear extremely complex phenomenon found in nature featuring the high sensitivity to the initial conditions. These features are defined as butterfly effect, the term coined by Lorenz in 1963 . Chaos theory is widely applicable to various fields of applied sciences and engineering, for instance, secure communication [1], biomedical engineering [2], machine learning, circuits [3], finance models [4], jerk systems [5], weather models [6], neural networks [7], oscillations [8], robotics [9], chemical reactions [10], encryption [11], ecological models [12], etc. Consequently, chaos theory has become one of the most appealing fields for researchers and engineers in recent times.

Historically, chaos theory dates back to the remarkable work of Poincare [13] established in 1890s while dealing with three-body problem to stabilize the solar system. In fact, he advocated the qualitatively behaviour, using geometric quantitatively to display the universal configuration of all solutions. Despite the observations made by Poincare [13], the first formal introduction of chaos in a deterministic system was proposed by Lorenz [14]. Lorenz observed that simple meteorological models depicted sensitive dependence on the initial conditions, known as butterfly effect, which established that chaos theory is widely applicable in other interdisciplinary areas also. Though the fundamental work on chaos synchronization had been carried by [15], the rapid growth in the field was seen after the phenomenal work of Ott et al. [16]. However, the concept of chaos synchronization was first introduced by Pecora and Carroll [17], wherein they performed synchronization of two identical chaotic systems based on master-slave composition which was unknown for the past 3 decades. Later on, many researchers carried forward the pioneered work of Pecora and Carroll and it has been established that chaos synchronization is also achievable for non-identical chaotic systems possessing absolutely different initial conditions.

A. Khan $\cdot$ H. Chaudhary $(\varangle)$

Department of Mathematics, Jamia Millia Islamia, New Delhi, India

E-mail: harindri20dbc@gmail.com

E-mail: akhan12@jmi.ac.in 
In the available literature, a variety of synchronization techniques have been introduced and executed on many chaotic systems to gain stability. These include complete synchronization [18], lag synchronization [19], anti-synchronization [20], hybrid synchronization [21], projective synchronization [22], hybrid projective synchronization [23], modified projective synchronization [24], combination synchronization [25], combination-combination (C-C) synchronization [26], compound synchronization [27] and so on.

Up to now, numerous chaos control techniques have been formulated to attain chaos control and synchronization, for example, active control [28], sliding mode control [29], adaptive control [30], optimal control [31], back stepping design [32], impulsive control [33], time-delay feedback control [34], etc.

A hyperchaotic (HC) system is characterized as a chaotic system having more than two positive Lyapunov exponents. Rossler [35] introduced the first classical HC system in the year 1979. Over the past few decades, a variety of classical HC systems have been emerged and investigated, for example, Lorenz system, Liu system, Chen system, Lu system, Cai system, Pehlivan system, Tigan system, Nikolov system, Sundarapandian system, Vaidyanathan system, etc.

Chaos synchronization among chaotic systems using adaptive control method (ACM) was introduced by Hubler [36] in 1989 for the first time. Synchronization of Rossler like system and Chua's circuit has been studied by using ACM in Ref. [37]. Also, adaptive control technique is studied to synchronize modified Chua's circuit system in Ref. [38]. Mainieri and Rehaceh firstly [39] formulated the notion of projective synchronization while synchronizing chaotic systems in 1999. In Ref. [1], projective synchronization and chaos in secure communication is studied. Further, in Ref. [40], complex projective synchronization between complex chaotic systems is discussed. Sun et al. [26] first proposed the $\mathrm{C}-\mathrm{C}$ synchronization scheme while synchronizing four identical or non-identical chaotic systems via active control method in 2012. A detailed study of $\mathrm{C}-\mathrm{C}$ synchronization between four non-identical chaotic systems with uncertain parameters using sliding mode control is described in Ref. [41]. Further in Ref. [42], C-C synchronization among four complex nonlinear chaotic systems is studied and also particular cases, for instance, projective synchronization and combination synchronization, are mentioned. Moreover, a generalized methodology of $\mathrm{C}-\mathrm{C}$ synchronization between $n$-dimensional chaotic fractional order nonlinear dynamical systems is developed in Ref. [43]. Here, the scheme discussed in Ref. [26] is extended to $\mathrm{C}-\mathrm{C}$ synchronization between four $n$-dimensional fractional order nonlinear chaotic systems. Also, complex $\mathrm{C}-\mathrm{C}$ synchronization among four newly constructed complex chaotic systems is discussed in Ref. [44]. In Ref. [45], chaos analysis and C-C synchronization of new HC systems having no equilibria are studied. Furthermore, $\mathrm{C}-\mathrm{C}$ synchronization is generalized to $n$-dimensional time-delay nonlinear chaotic systems using robust adaptive sliding mode control in Ref. [46]. Also, in Ref. [47], C-C anti-synchronization between four identical fractional order $\mathrm{HC}$ systems is discussed. Further, $\mathrm{C}-\mathrm{C}$ phase synchronization between different fractional order complex nonlinear chaotic systems via active control is studied in Ref. [48]. Also, finite-time combination synchronization among three non-identical chaotic systems is discussed in Ref. [49]. Moreover, combination synchronization between three different order chaotic systems via active backstepping technique is studied in [50].

Considering the aforementioned studies about $\mathrm{C}-\mathrm{C}$ synchronization, our paper aims to investigate a hybrid projective combination-combination synchronization (HPCCS) among four non-identical HC systems via ACM. Moreover, based on (LST), we formulate an adaptive control law (ACL) with an estimated parameter update law. Our approach has potentiality and is advantageous. If chaos synchronization can be controlled efficiently, then it may be easily possible to achieve significantly higher quality achievements for information processing and secure communications. The HPCCS scheme among four non-identical HC systems of integer order using ACM has not yet been explored till now. This shows the novelty of this presented research work.

Our studies are structured as follows: in Sect. 2, some necessary definitions and basic notations are presented to be used within this paper. Section 3 deals with the methodology of ACM. Section 4 contains the basic structured features of the considered HC systems. Section 5 investigates the HPCCS scheme via ACM strategy. Numerical simulations are executed to verify the obtained analytical results of the proposed scheme in Sect. 6 . Also, a comparative analysis has been discussed. Lastly, some conclusions are drawn in Sect. 7

\section{Preliminaries}

In this section, we initially describe the procedure to achieve $\mathrm{C}-\mathrm{C}$ synchronization according to master-slave configuration among four non-identical integer order HC systems with two master systems and two slave systems which are requisites for the subsequent sections. 
The two master systems and two slave systems are expressed as

$$
\begin{aligned}
& \dot{x_{1}}=f_{1}\left(x_{1}\right) \eta_{1}+F_{1}\left(x_{1}\right), \\
& \dot{x_{2}}=f_{2}\left(x_{2}\right) \eta_{2}+F_{2}\left(x_{2}\right), \\
& \dot{y_{1}}=g_{1}\left(y_{1}\right) \theta_{1}+G_{1}\left(y_{1}\right)+u_{1}, \\
& \dot{y_{2}}=g_{2}\left(y_{2}\right) \theta_{2}+G_{2}\left(y_{2}\right)+u_{2},
\end{aligned}
$$

where $x_{1}=\left(x_{11}, x_{12}, \ldots, x_{1 n}\right)^{T} \in R^{n}, x_{2}=\left(x_{21}, x_{22}, \ldots, x_{2 n}\right)^{T} \in R^{n}, y_{1}=\left(y_{11}, y_{12}, \ldots, y_{1 n}\right)^{T} \in R^{n}$, $y_{2}=\left(y_{21}, y_{22}, \ldots, y_{2 n}\right)^{T} \in R^{n}$ are the state vectors of master and slave systems (1), (2), (3) and (4), respectively, $f_{1}, f_{2}, g_{1}, g_{2}: R^{n} \rightarrow R^{n}$ are four nonlinear continuous functions, $\eta_{1}=\left(\eta_{11}, \eta_{12}, \ldots, \eta_{1 p_{1}}\right)^{T}$ is a $p_{1} \times 1$ unknown parameter vector of the first master system $(1), \eta_{2}=\left(\eta_{21}, \eta_{22}, \ldots, \eta_{2 p_{2}}\right)^{T}$ is a $p_{2} \times 1$ unknown parameter vector of the second master system $(2), \theta_{1}=\left(\theta_{11}, \theta_{1} 2, \ldots, \theta_{1 p_{3}}\right)^{T}$ is a $p_{3} \times 1$ unknown parameter vector of the first slave system (3), $\theta_{2}=\left(\theta_{21}, \theta_{22}, \ldots, \theta_{2 p_{3}}\right)^{T}$ is a $p_{4} \times 1$ unknown parameter vector of the second slave system (4), $u_{1}=\left(u_{11}, u_{12}, \ldots, u_{1 n}\right) \in R^{n}, u_{2}=\left(u_{21}, u_{22}, \ldots, u_{2 n}\right) \in R^{n}$ are the suitable controllers which are to be designed.

Definition 2.1 If there exist four constant matrices $P, Q, R, S \in R^{n} \times R^{n}$ and $R \neq 0$ or $S \neq 0$ such that

$$
\lim _{t \rightarrow \infty}\|e\|=\lim _{t \rightarrow \infty}\left\|\left(P x_{1}+Q x_{2}-R y_{1}-S y_{2}\right)\right\|=0,
$$

the combination of two master systems (1) and (2) is said to perform C-C synchronization with the combination of two slave systems (3) and (4) and $\|$.$\| denotes the matrix norm.$

Remark 2.2 The constant matrices $P, Q, R$ and $S$ are called the scaling matrices. In addition, $P, Q, R, S$ can be extended as matrices of functions of state variables $x_{1}, x_{2}, y_{1}$ and $y_{2}$.

Remark 2.3 If $R=0$ or $S=0$, then C-C synchronization problem turns out to be the combination synchronization problem.

Remark 2.4 The combination synchronization problem will be converted into the chaos control problem for $P=Q=R=0$ or $P=Q=S=0$.

Remark 2.5 If $R=S=-I$ and $P=Q=-\alpha I$, for $\alpha=1$ it turns into C-C complete synchronization and for $\alpha=-1$ it will be reduced to $\mathrm{C}-\mathrm{C}$ anti-synchronization. Combination of complete and anti-synchronization makes HPCCS. Thus, the HPCCS error takes the form:

$$
e=\left(y_{1}+y_{2}\right)-\alpha_{i}\left(x_{1}+x_{2}\right),
$$

where $\alpha=\operatorname{diag}\left(\alpha_{1}, \alpha_{2}, \ldots, \alpha_{n}\right)$.

Remark 2.6 Definition 2.1 displays that the combination of master systems and slave systems can be expanded to three or more chaotic systems. Further, master systems and slave systems of the $\mathrm{C}-\mathrm{C}$ synchronization scheme can be identical or non-identical.

\section{Methodology of adaptive control method}

Consider the chaotic (or HC) master system and the corresponding chaotic (or HC) slave system as

$$
\begin{aligned}
& \dot{x}=f_{1}(x)+f_{2}(x) \eta, \\
& \dot{y}=g_{1}(y)+g_{2}(y) \beta+u,
\end{aligned}
$$

where $x=\left(x_{1}, x_{2}, \ldots, x_{n}\right)^{T}, y=\left(y_{1}, y_{2}, \ldots, y_{n}\right)^{T}$ are the state vectors, $u=\left(u_{i}, i=1,2, \ldots, n\right) \in R^{n}$ is the controller vector , $f_{1}, g_{1}: R^{n} \rightarrow R^{n}$ are non-linear continuous vector functions, $f_{2}: R^{n} \rightarrow R^{n \times m_{1}}$, $g_{2}: R^{n} \rightarrow R^{n \times m_{2}}$ are matrix. $\eta \in R^{m_{1}}$, and $\beta \in R^{m_{2}}$ are non-linear parameter vectors.

Suppose error is described as

$$
e(t)=y(t)-x(t),
$$


where $e(t)=\left(e_{i} ; i=1,2, \ldots, n\right)^{T}$ is the error function and $\|\cdot\|$ represents the vector norm.

It follows that

$$
\dot{e}(t)=\dot{y}(t)-\dot{x}(t) .
$$

Using (6) and (7), the error dynamics becomes

$$
\dot{e}(t)=g_{1}(y)+g_{2}(y) \beta+u-f_{1}(x)-f_{2}(x) \eta .
$$

Now, we design appropriate control function $u$ and parameter update laws accordingly to ensure that master and slave systems with unknown parameters approach to desired synchronization state.

For that the controller is chosen as

$$
u=-g_{1}(y)-g_{2}(y) \hat{\beta}+f_{1}(x)+f_{2}(x) \hat{\eta}-k e,
$$

where $\hat{\eta}(t), \hat{\beta}(t)$ are the estimated values of unknown parameter vectors, respectively, and $k$ is an arbitrarily chosen positive number known as gain constant.

The parameter update laws are described as

$$
\begin{aligned}
& \dot{\hat{\eta}}=-\left[f_{2}(x)\right]^{T} e+k_{\eta} \tilde{\eta} \\
& \dot{\hat{\beta}}=\left[g_{2}(y)\right]^{T} e+k_{\beta} \tilde{\beta},
\end{aligned}
$$

where $\tilde{\eta}=\eta-\hat{\eta}$ and $\tilde{\beta}=\beta-\hat{\beta}$.

Choosing the Lyapunov function as

$$
V(t)=\frac{1}{2}\left(e^{T} e+\tilde{\eta}^{T} \tilde{\eta}+\tilde{\beta}^{T} \tilde{\beta}\right) .
$$

The derivative of $V$ is written as

$$
\begin{aligned}
\dot{V}(t) & =e^{T} \dot{e}+\tilde{\eta}^{T}(-\dot{\hat{\eta}})+\tilde{\beta}^{T}(-\dot{\hat{\beta}}) \\
& =e^{T}\left[g_{2}(y) \tilde{\beta}-f_{2}(x) \tilde{\eta}-k e\right]-\tilde{\eta}^{T}\left[-\left[f_{2}(x)\right]^{T} e+k_{\eta} \tilde{\eta}\right]-\tilde{\beta}^{T}\left[\left[g_{2}(y)\right]^{T} e+k_{\beta} \tilde{\beta}\right] \\
& =e^{T} g_{2}(y) \tilde{\beta}-e^{T} f_{2}(x) \tilde{\eta}-k e^{T} e+\tilde{\eta}^{T}\left[f_{2}(x)\right]^{T} e-k_{\eta} \tilde{\eta}^{T} \tilde{\eta}-\tilde{\beta}^{T}\left[g_{2}(y)\right]^{T} e-k_{\beta} \tilde{\beta}^{T} \tilde{\beta} \\
& =-k e^{T} e-k_{\eta} \tilde{\eta}^{T} \tilde{\eta}-k_{\beta} \tilde{\beta}^{T} \tilde{\beta}-\left[\tilde{\eta}^{T}\left[f_{2}(x)\right]^{T} e\right]^{T}+\tilde{\eta}^{T}\left[f_{2}(x)\right]^{T} e+\left[\tilde{\beta}^{T}\left[g_{2}(y)\right]^{T} e\right]^{T}-\tilde{\beta}^{T}\left[g_{2}(y)\right]^{T} e \\
& =-k e^{T} e-k_{\eta} \tilde{\eta}^{T} \tilde{\eta}-k_{\beta} \tilde{\beta}^{T} \tilde{\beta} \\
& <0,
\end{aligned}
$$

which establishes that $\dot{V}$ is negative definite.

In accordance with LST, the error dynamical system is globally and asymptotically stable in the neighbourhood of the equilibrium points.

\section{System description}

Wang et al. [51] introduced the HC system:

$$
\begin{aligned}
\dot{x}_{11} & =a_{1}\left(y_{11}-x_{11}\right)+w_{11} \\
\dot{y}_{11} & =c_{1} x_{11}-y_{11}-x_{11} z_{11} \\
\dot{z}_{11} & =x_{11} y_{11}-b_{1} z_{11} \\
\dot{w}_{11} & =y_{11} z_{11}+d_{1} w_{11},
\end{aligned}
$$

where $x_{11}, y_{11}, z_{11}, w_{11}$ are the states variables for the system (8) and for the system parameters $a_{1}=10, b_{1}=$ $8 / 3, c_{1}=28, d_{1}=-1$, the system (8) displays HC behaviour as discussed in Ref. [51].

Lu et al. [52] announced the HC system: 


$$
\begin{aligned}
\dot{x}_{21} & =a_{2}\left(y_{21}-x_{21}\right)+w_{21} \\
\dot{y}_{21} & =-x_{21} z_{21}+c_{2} y_{21} \\
\dot{z}_{21} & =x_{21} y_{21}-b_{2} z_{21} \\
\dot{w}_{21} & =x_{21} z_{21}+d_{2} w_{21},
\end{aligned}
$$

where $x_{21}, y_{21}, z_{21}, w_{21}$ are the state variables for the system (9) and for the system parameters $a_{2}=36, b_{2}=$ $3, c_{2}=20, d_{2}=1.3$, the system (9) exhibits HC behaviour as proven in Ref. [52].

Zheng et al. [53] proposed the HC system:

$$
\begin{aligned}
\dot{x}_{31} & =a_{3}\left(y_{31}-x_{31}\right)+w_{31} \\
\dot{y}_{31} & =b_{3} x_{31}+x_{31} z_{31}+c_{3} y_{31}+w_{31} \\
\dot{z}_{31} & =-x_{31} x_{31}-d_{3} z_{31} \\
\dot{w}_{31} & =-h_{3} y_{31},
\end{aligned}
$$

where $x_{31}, y_{31}, z_{31}, w_{31}$ are the state variables for system (10) and for the system parameters $a_{3}=20, b_{3}=$ $14, c_{3}=10.6, d_{3}=2.8, h_{3}=4$, the system (10) shows HC behaviour as studied in Ref. [53].

Wei et al. [54] found the HC system:

$$
\begin{aligned}
\dot{x}_{41} & =a_{4}\left(y_{14}-x_{14}\right) \\
\dot{y}_{41} & =-x_{41} z_{41}-c_{4} y_{41}+d_{4} w_{41} \\
\dot{z}_{41} & =b_{4}+x_{41} y_{41} \\
\dot{w}_{41} & =-h_{4} y_{41},
\end{aligned}
$$

where $x_{41}, y_{41}, z_{41}, w_{41}$ are the state variables for the system (11) and for the system parameters $a_{4}=10, b_{4}=$ 25, $c_{4}=-2.5, d_{4}=1, h_{4}=1$, system (11) depicts HC behaviour as shown in Ref. [54]. In addition, Fig. 1 and Fig. 2 display 2-D and 3-D phase portraits respectively of the aforementioned four hyperchaotic systerms.

In the next section, we shall present the HPCCS scheme using ACM.

\section{Illustrative example}

In this section, we discuss the ACL and the parameter estimation update law to stabilize the HC systems. For convenience, the systems (8) and (9) are treated as the master systems and the systems (10) and (11) are treated as the slave systems with controllers may be written as

$$
\begin{aligned}
& \dot{x}_{31}=a_{3}\left(y_{31}-x_{31}\right)+w_{31}+u_{11} \\
& \dot{y}_{31}=b_{3} x_{31}+x_{31} z_{31}+c_{3} y_{31}+w_{31}+u_{12} \\
& \dot{z}_{31}=-x_{31} x_{31}-d_{3} z_{31}+u_{13} \\
& \dot{w}_{31}=-h_{3} y_{31}+u_{14} \\
& \dot{x}_{41}=a_{4}\left(y_{14}-x_{14}+u_{21}\right. \\
& \left.\dot{y}_{41}=-x_{41} z_{41}-c_{4} y_{41}\right)+d_{4} w_{41}+u_{22} \\
& \dot{z}_{41}=b_{4}+x_{41} y_{41}+u_{23} \\
& \dot{w}_{41}=-h_{4} y_{41}+u_{24},
\end{aligned}
$$

where $u_{11}, u_{12}, u_{13}, u_{14}, u_{21}, u_{22}, u_{23}, u_{24}$ are the adaptive control inputs to be designed using ACM.

We define the HPCCS errors as

$$
\begin{aligned}
& e_{11}=x_{41}+x_{31}-\alpha_{1}\left(x_{11}+x_{21}\right) \\
& e_{12}=y_{41}+y_{31}-\alpha_{2}\left(y_{11}+y_{21}\right) \\
& e_{13}=z_{41}+z_{31}-\alpha_{3}\left(z_{11}+z_{21}\right) \\
& e_{14}=w_{41}+w_{31}-\alpha_{4}\left(w_{11}+w_{21}\right) .
\end{aligned}
$$

The HPCCS error dynamics takes the form: 


$$
\begin{aligned}
& \dot{e_{11}}=\dot{x_{41}}+\dot{x_{31}}-\alpha_{1}\left(\dot{x_{11}}+\dot{x_{21}}\right) \\
& \dot{e}_{12}=\dot{y_{41}}+\dot{y_{31}}-\alpha_{2}\left(\dot{y_{11}}+\dot{y_{21}}\right) \\
& \dot{e_{13}}=z_{41}+z_{31}-\alpha_{3}\left(z_{11}+z_{21}\right) \\
& \dot{e_{14}}=\dot{w_{41}}+\dot{w_{31}}-\alpha_{4}\left(\dot{w_{11}}+\dot{w_{21}}\right) .
\end{aligned}
$$

Substituting the equations of master systems (8)-(9) and slave systems (12)-(13) in (15), the error dynamics transforms to

$$
\begin{aligned}
\dot{e_{11}=} & a_{3}\left(y_{31}-x_{31}\right)+w_{31}-\alpha_{1} a_{1}\left(y_{11}-x_{11}\right)-\alpha_{1} w_{11}+a_{4}\left(y_{41}-x_{41}\right) \\
& -\alpha_{1} a_{2}\left(y_{21}-x_{21}\right)-\alpha_{1} w_{21}+u_{11}+u_{21} \\
e_{12}= & b_{3} x_{31}+x_{31} z_{31}+c_{3} y_{31}+w_{31}-\alpha_{2}\left(c_{1} x_{11}-y_{11}-x_{11} z_{11}\right) \\
& -x_{41} z_{41}-c_{4} y_{41}+d_{4} w_{41}-\alpha_{2}\left(-x_{21} z_{21}+c_{2} y_{21}\right)+u_{12}+u_{22} \\
e_{13}= & -x_{31} x_{31}-d_{3} z_{31}-\alpha_{3}\left(x_{11} y_{11}-b_{1} z_{11}\right) \\
& -b_{4}+x_{41} y_{41}-\alpha_{3}\left(x_{21} y_{21}-b_{2} z_{21}\right)+u_{13}+u_{23} \\
e_{14}= & -h_{3} y_{31}-\alpha_{4}\left(-y_{11} z_{11}+d_{1} w_{11}\right)-h_{4} y_{41} \\
& -\alpha_{4}\left(x_{21} z_{21}+d_{2} w_{21}\right)+u_{14}+u_{24} .
\end{aligned}
$$

The adaptive controllers are defined as

$$
\begin{aligned}
u_{11}+u_{21}= & -\hat{a_{3}}\left(y_{31}-x_{31}\right)-w_{31}+\alpha_{1} \hat{a_{1}}\left(y_{11}-x_{11}\right)+\alpha_{1} w_{11}-k_{1} e_{11} \\
& -\hat{a_{4}}\left(y_{41}-x_{41}\right)+\hat{\alpha}_{1} \hat{a_{2}}\left(y_{21}-x_{21}\right)+\alpha_{1} w_{21} \\
u_{12}+u_{22}= & -\hat{b_{3}} x_{31}-x_{31} z_{31}-\hat{c_{3}} y_{31}-w_{31}+\alpha_{2}\left(\hat{c_{1}} x_{11}-y_{11}-x_{11} z_{11}\right) \\
& -k_{2} e_{12}+x_{41} z_{41}+\hat{c_{4}} y_{41}-\hat{d_{4}} w_{41}+\alpha_{2}\left(-x_{21} z_{21}+\hat{c_{2}} y_{21}\right) \\
u_{13}+u_{23}= & x_{31} x_{31}+\hat{d_{3}} z_{31}+\alpha_{3}\left(x_{11} y_{11}-\hat{b_{1}} z_{11}\right)-k_{3} e_{13}+\hat{b_{4}}-x_{41} y_{41} \\
& +\alpha_{3}\left(x_{21} y_{21}-\hat{b_{2}} z_{21}\right) \\
u_{14}+u_{24}= & \hat{h_{3}} y_{31}+\alpha_{4}\left(-y_{11} z_{11}+\hat{d_{1}} w_{11}\right)-k_{4} e_{14}+\hat{h_{4}} y_{41} \\
& +\alpha_{4}\left(x_{21} z_{21}+\hat{d_{2}} w_{21}\right),
\end{aligned}
$$

where $k_{1}, k_{2}, k_{3}$ and $k_{4}$ are positive gain constants.

Putting the values of controllers (17) in error dynamics (16), we get

$$
\begin{aligned}
\dot{e i 1}= & \left(a_{3}-\hat{a}_{3}\right)\left(y_{31}-x_{31}\right)-\alpha_{1}\left(a_{1}-\hat{a}_{1}\right)\left(y_{11}-x_{11}\right)+\left(a_{4}-\hat{a}_{4}\right)\left(y_{41}-x_{41}\right) \\
& -\alpha_{1}\left(a_{2}-\hat{a}_{2}\right)\left(y_{21}-x_{21}\right)-k_{1} e_{11} \\
e_{12}= & \left(b_{3}-\hat{b}_{3}\right) x_{31}+\left(c_{3}-\hat{c}_{3}\right) y_{31}-\alpha_{2}\left(c_{1}-\hat{c}_{1}\right) x_{11}-\left(c_{4}-\hat{c}_{4}\right) y_{41} \\
& +\left(d_{4}-\hat{d}_{4}\right) w_{41}-\alpha_{2}\left(c_{2}-\hat{c}_{2}\right) y_{21}-k_{2} e_{12} \\
e_{13}= & -\left(d_{3}-\hat{d}_{3}\right) z_{31}+\alpha_{3}\left(b_{1}-\hat{b}_{1}\right) z_{11}-\left(b_{4}-\hat{b}_{4}\right)+\alpha_{3}\left(b_{2}-\hat{b}_{2}\right) z_{21} \\
& -k_{3} e_{13} \\
e_{14}= & -\left(h_{3}-\hat{h}_{3}\right) y_{31}-\alpha_{4}\left(d_{1}-\hat{d}_{1}\right) w_{11}-\left(h_{4}-\hat{h}_{4}\right) y_{41}-\alpha_{4}\left(d_{2}-\hat{d}_{2}\right) w_{21} \\
& -k_{4} e_{14} .
\end{aligned}
$$

We define parameter estimation error as follows:

$$
\begin{aligned}
& \tilde{a_{1}}=a_{1}-\hat{a_{1}}, \tilde{a_{2}}=a_{2}-\hat{a_{2}}, \tilde{a_{3}}=a_{3}-\hat{a_{3}}, \tilde{a_{4}}=a_{4}-\hat{a_{4}}, \tilde{b_{1}}=b_{1}-\hat{b_{1}}, \tilde{b_{2}}=b_{2}-\hat{b_{2}}, \\
& \tilde{b_{3}}=b_{3}-\hat{b_{3}}, \tilde{b_{4}}=b_{4}-\hat{b_{4}} \tilde{c_{1}}=c_{1}-\hat{c_{1}}, \tilde{c_{2}}=c_{2}-\hat{c_{2}}, \tilde{c_{3}}=c_{3}-\hat{c_{3}}, \tilde{c_{4}}=c_{4}-\hat{c_{4}}, \\
& \tilde{d}_{1}=d_{1}-\hat{d_{1}}, \tilde{d_{2}}=d_{2}-\hat{d_{2}}, \tilde{d_{3}}=d_{3}-\hat{d_{3}}, \tilde{d_{4}}=d_{4}-\hat{d}, \tilde{h_{3}}=h_{3}-\hat{h_{3}}, \tilde{h_{4}}=h_{4}-\hat{h_{4}} .
\end{aligned}
$$

From Eqs. (18) and (19), we obtain 


$$
\begin{aligned}
\dot{e}_{11}= & \tilde{a_{3}}\left(y_{31}-x_{31}\right)-\alpha_{1} \tilde{a_{1}}\left(y_{11}-x_{11}\right)+\tilde{a_{4}}\left(y_{41}-x_{41}\right) \\
& -\alpha_{1} \tilde{a_{2}}\left(y_{21}-x_{21}\right)-k_{1} e_{11} \\
\dot{e}_{12}= & \tilde{b_{3}} x_{31}+\tilde{c_{3}} y_{31}-\alpha_{2} \tilde{c_{1}} x_{11}-\tilde{c_{4}} y_{41}+\tilde{d}_{4} w_{41}-\alpha_{2} \tilde{c_{2}} y_{21}-k_{2} e_{12} \\
e_{13}= & -\tilde{d}_{3} z_{31}+\alpha_{3} \tilde{b_{1}} z_{11}-\tilde{b_{4}}+\alpha_{3} \tilde{b_{2}} z_{21}-k_{3} e_{13} \\
\dot{e}_{14}= & -\tilde{h_{3}} y_{31}-\alpha_{4} \tilde{d}_{1} w_{11}-\tilde{h_{4}} y_{41}-\alpha_{4} \tilde{d}_{2} w_{21}-k_{4} e_{14} .
\end{aligned}
$$

Next, we consider the candidate for Lyapunov function given by

$$
\begin{aligned}
V= & \frac{1}{2}\left[e_{11}^{2}+{\tilde{e_{12}}}^{2}+{\tilde{e_{13}}}^{2}+{e_{14}^{2}}^{2}+{\tilde{a_{1}}}^{2}+{\tilde{a_{2}}}^{2}+{\tilde{a_{3}}}^{2}+{\tilde{a_{4}}}^{2}\right. \\
& +{\tilde{b_{1}}}^{2}+{\tilde{b_{2}}}^{2}+{\tilde{b_{3}}}^{2}+{\tilde{b_{4}}}^{2}+{\tilde{c_{1}}}^{2}+{\tilde{c_{2}}}^{2}+{\tilde{c_{3}}}^{2} \\
& \left.+{\tilde{c_{4}}}^{2}+{\tilde{d_{1}}}^{2}+{\tilde{d_{2}}}^{2}+{\tilde{d_{3}}}^{2}+{\tilde{d_{4}}}^{2}+{\tilde{h_{3}}}^{2}+{\tilde{h_{4}}}^{2}\right],
\end{aligned}
$$

which is a positive definite function. Differentiating $V$ along the trajectories of (21), we obtain

$$
\begin{aligned}
& \dot{V}=e_{11} \dot{e}_{11}+e_{12} e_{12}+e_{13} e_{13}+e_{14} e_{14}-\tilde{a_{1}} \dot{\hat{a}_{1}}-\tilde{a_{2}} \dot{\hat{a_{2}}}-\tilde{a_{3}} \dot{\hat{a_{3}}}-\tilde{a_{4}} \dot{\hat{a_{4}}} \\
& -\tilde{b_{1}} \dot{\hat{b_{1}}}-\tilde{b_{2}} \dot{\hat{b_{2}}}-\tilde{b_{3}} \dot{\hat{b_{3}}}-\tilde{b_{4}} \dot{\hat{b_{4}}}-\tilde{c_{1}} \dot{\hat{c_{1}}} \\
& -\tilde{c_{2}} \dot{\hat{c}_{2}}-\tilde{c_{3}} \dot{\hat{c_{3}}}-\tilde{c_{4}} \dot{\hat{c}_{4}}-\tilde{d}_{1} \dot{\hat{d}}_{1} \\
& -\tilde{d}_{2} \dot{\hat{d}}_{2}-\tilde{d}_{3} \dot{\hat{d}}_{3}-\tilde{d}_{4} \dot{\hat{d}}_{4}-\tilde{h}_{3} \dot{\hat{h}}_{3}-\tilde{h}_{4} \dot{\hat{h_{4}}} \text {. }
\end{aligned}
$$

In view of (22), we define the parameter estimation laws as follows:

$$
\begin{aligned}
& \dot{\hat{a_{1}}}=-\alpha_{1}\left(y_{11}-x_{11}\right) e_{11}+k_{5} \tilde{a}_{1} \\
& \dot{\hat{a_{2}}}=-\alpha_{1}\left(y_{21}-x_{21}\right) e_{11}+k_{6} \tilde{a}_{2} \\
& \dot{\hat{a_{3}}}=\left(y_{31}-x_{31}\right) e_{11}+k_{7} \tilde{a}_{3} \\
& \dot{\hat{a}_{4}}=\left(y_{41}-x_{41}\right) e_{11}+k_{8} \tilde{a}_{4} \\
& \dot{\hat{b_{1}}}=\alpha_{3} z_{11} e_{13}+k_{9} \tilde{b}_{1} \\
& \dot{\hat{b_{2}}}=\alpha_{3} z_{12} e_{13}+k_{10} \tilde{b}_{2} \\
& \dot{\hat{b_{3}}}=x_{31} e_{12}+k_{11} \tilde{b}_{3} \\
& \dot{\hat{b_{4}}}=-e_{13}+k_{12} \tilde{b}_{4} \\
& \dot{\hat{c_{1}}}=-\alpha_{2} x_{11} e_{12}+k_{13} \tilde{c}_{1} \\
& \dot{\hat{c_{2}}}=-\alpha_{2} y_{21} e_{12}+k_{14} \tilde{c}_{2} \\
& \dot{\hat{c_{3}}}=y_{31} e_{12}+k_{15} \tilde{c}_{3} \\
& \dot{\hat{c_{4}}}=-y_{41} e_{12}+k_{16} \tilde{c}_{4} \\
& \dot{\dot{d}_{1}}=\alpha_{4} w_{11} e_{14}+k_{17} \tilde{d}_{1} \\
& \dot{\hat{d}_{2}}=\alpha_{4} w_{12} e_{14}+k_{18} \tilde{d}_{2} \\
& \dot{\hat{d}_{3}}=-z_{31} e_{13}+k_{19} \tilde{d}_{3} \\
& \dot{\hat{d}_{4}}=w_{41} e_{12}+k_{20} \tilde{d}_{4} \\
& \dot{\hat{h_{3}}}=-y_{31} e_{14}+k_{21} \tilde{h}_{3} \\
& \dot{\hat{h_{4}}}=-y_{41} e_{14}+k_{22} \tilde{h}_{4} .
\end{aligned}
$$




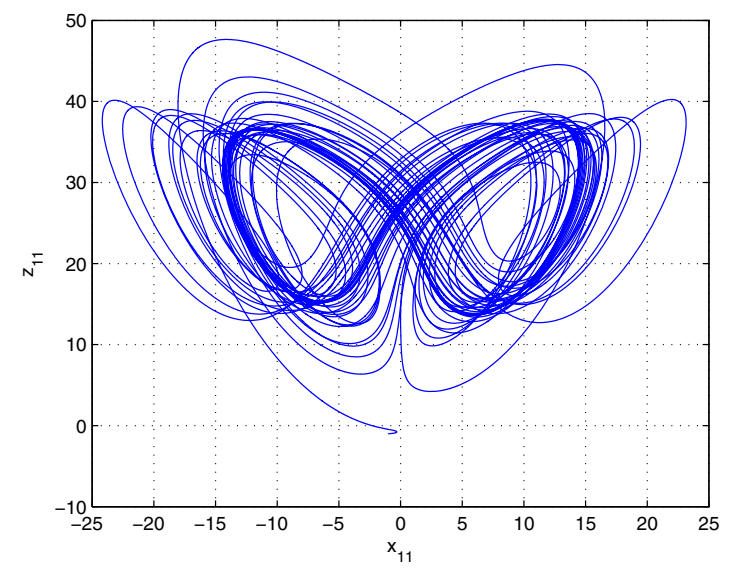

(a)



(c)

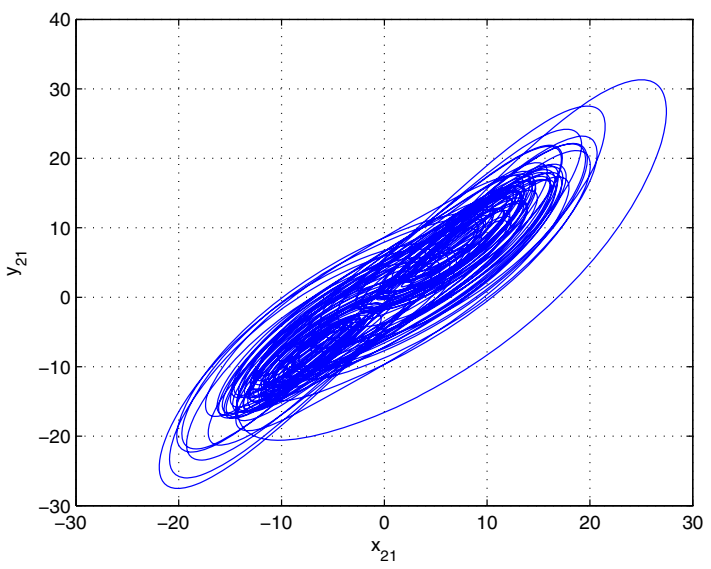

(b)

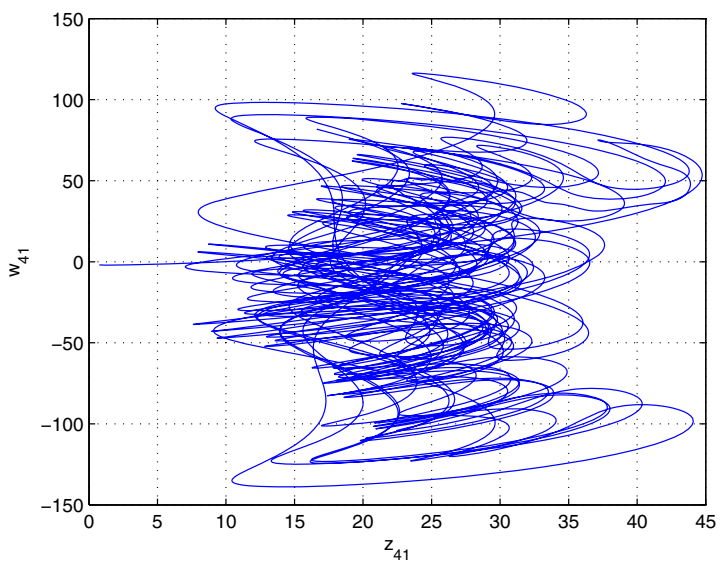

(d)

Fig. 1 Phase portraits of hyperchaotic systems a $x_{11}-z_{11}$-plane, $\mathbf{b} x_{21}-y_{21}$-plane, $\mathbf{c} x_{31}-z_{31}$-plane, $\mathbf{d} z_{41}-w_{41}$-plane

Theorem 5.1 The non-identical HC systems (8)-(9) and (12)-(13) are globally and asymptotically hybrid projective $C-C$ synchronized for all initial states under the defined $A C L s$ (17) and the parameter update law (23), where $k_{i}, i=1,2,3 \ldots, 22$ are positive gain constants.

Proof The Lyapunov function $V$ defined by Eq. (21) is a positive definite function. Inserting the error dynamics (20) and using parameter update laws (23) into (22), the time derivative of $V$ is obtained as

$$
\begin{aligned}
\dot{V}= & -k_{1} e_{11}^{2}-k_{2} e_{12}^{2}-k_{3} e_{13}^{2}-k_{4} e_{14}^{2}-k_{5}{\tilde{a_{1}}}^{2}-k_{6}{\tilde{a_{2}}}^{2} \\
& -k_{7}{\tilde{a_{3}}}^{2}-k_{8}{\tilde{a_{4}}}^{2}-k_{9}{\tilde{b_{1}}}^{2}-k_{1} 0{\tilde{b_{2}}}^{2}-k_{1} \tilde{b}_{3}{ }^{2}-k_{1} 2{\tilde{b_{4}}}^{2} \\
& -k_{13}{\tilde{c_{1}}}^{2}-k_{14}{\tilde{c_{2}}}^{2}-k_{14}{\tilde{c_{3}}}^{2}-k_{15}{\tilde{c_{4}}}^{2}-k_{16}{\tilde{d_{1}}}^{2} \\
& -k_{17}{\tilde{d_{2}}}^{2}-k_{18}{\tilde{d_{3}}}^{2}-k_{19}{\tilde{d_{4}}}^{2}-k_{20}{\tilde{h_{3}}}^{2}-k_{21}{\tilde{h_{4}}}^{2} .
\end{aligned}
$$

This shows that $\dot{V}<0$ which ensures that $\dot{V}$ is negative definite in $\mathrm{R}^{22}$.

Thus, according to LST, we deduce that the HPCCS error $e(t) \rightarrow 0$ asymptotically as $t \rightarrow \infty$ for all initial conditions $e(0) \in R^{4}$. Hence, the proof is complete. 


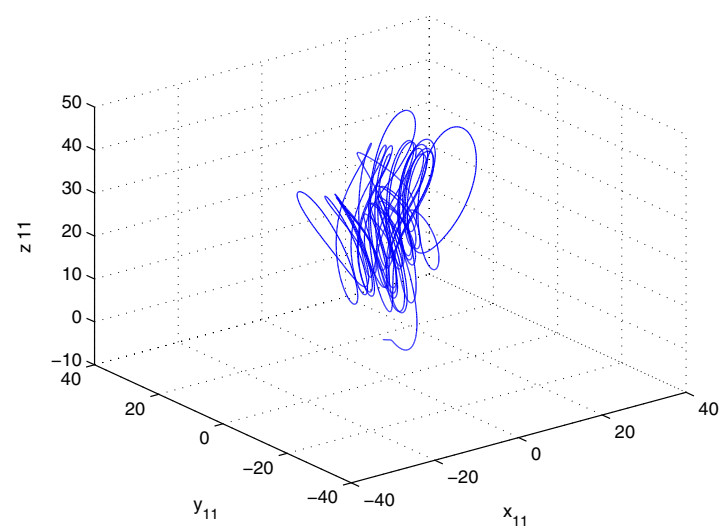

(a)

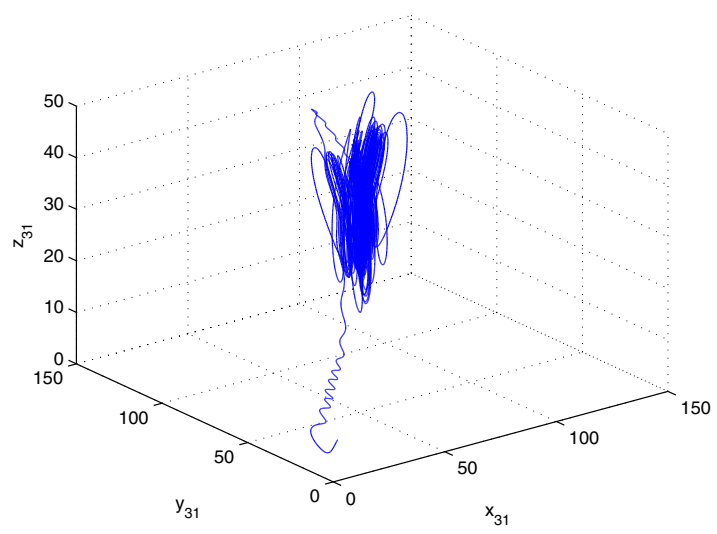

(c)

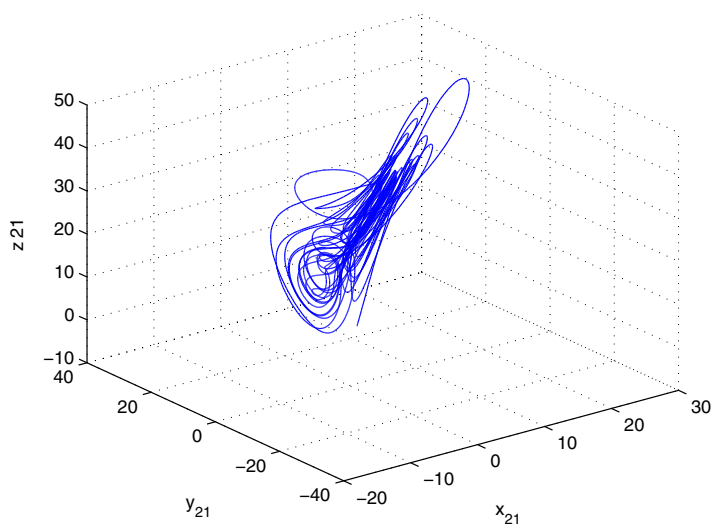

(b)

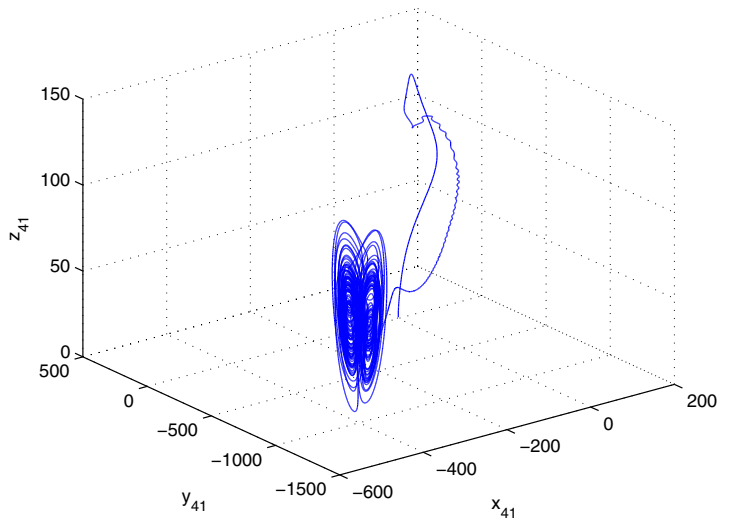

(d)

Fig. 2 Phase portraits of hyperchaotic systems in a $x_{11}-y_{11}-z_{11}$ space, $\mathbf{b} x_{21}-y_{21}-z_{21}$ space, $\mathbf{c} x_{31}-y_{31}-z_{31}$ space, d $x_{41}-y_{41}-z_{41}$ space

\section{Numerical simulation}

In this section, we demonstrate the numerical simulations in order to check the effectivity and feasibility of the investigated approach. To achieve this, fourth order Runge-Kutta method has been used. For the simulation procedure, the parameter values are taken as $a_{1}=10, b_{1}=8 / 3, c_{1}=28, d_{1}=-1, a_{2}=$ $36, b_{2}=3, c_{2}=20, d_{2}=1.3, a_{3}=20, b_{3}=14, c_{3}=10.6, d_{3}=2.8, h_{3}=4, a_{4}=10, b_{4}=25$, $c_{4}=-2.5, d_{4}=1, h_{4}=1$. The initial conditions for the master systems and slave systems are arbitrarily chosen as $\left(x_{11}(0), y_{11}(0), z_{11}(0), w_{11}(0)\right)=(-1,1,-1,1),\left(x_{21}(0), y_{21}(0), z_{21}(0), w_{21}(0)\right)=(5,8,-1,-3)$, $\left(x_{31}(0), y_{31}(0), z_{31}(0), w_{31}(0)\right)=(5,4,7,2),\left(x_{41}(0), y_{41}(0), z_{41}(0), w_{41}(0)\right)=(0.2,0.1,0.75,-2)$. The control gains are selected as $k_{i}=4$ for $i=1,2, \ldots, 22$.

Case 1. Let us assume the scaling matrix $\alpha$ with $\alpha_{1}=\alpha_{2}=\alpha_{3}=1$. In this case, we achieve complete C-C synchronization with master systems (8)-(9) and slave systems (12)-(13). The trajectories of master and slave systems with the control inputs are depicted in Fig. 3a-d. Further, initial states for error system are obtained as $\left(e_{11}, e_{12}, e_{13}, e_{14}\right)=(1.2,-4.9,9.75,2)$. The convergence of synchronization error $e(t)$ to zero as $t$ approaches infinity as displayed in Fig. 3e shows that the HPCCS among two master systems and two slave systems has been achieved via ACM. Moreover, Fig. 3f displays that the estimated parameters converge to their respective original values asymptotically with time. Thus, the proposed HPCCS scheme between master and slave systems is verified computationally.

Case 2. When the scaling matrix $\alpha$ is taken as $\alpha_{1}=\alpha_{2}=\alpha_{3}=\alpha_{4}=-1$, then we achieve C-C antisynchronization between the master systems (8)-(9) and the slave systems (12)-(13). Figure 4a-d exhibits 


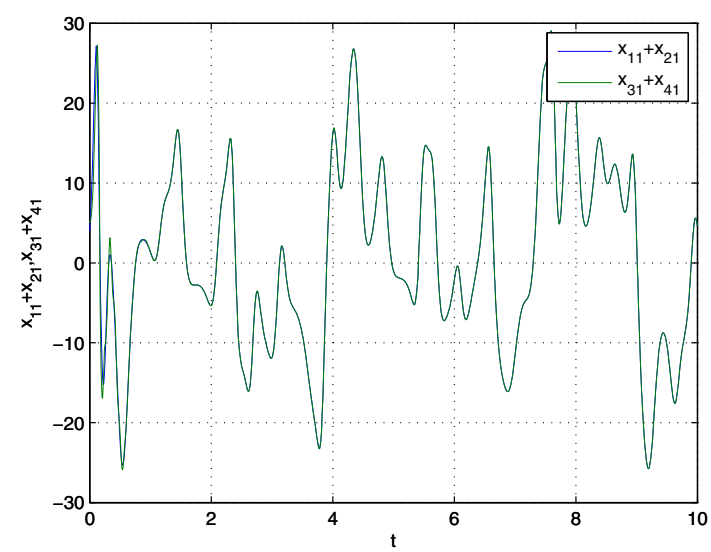

(a)

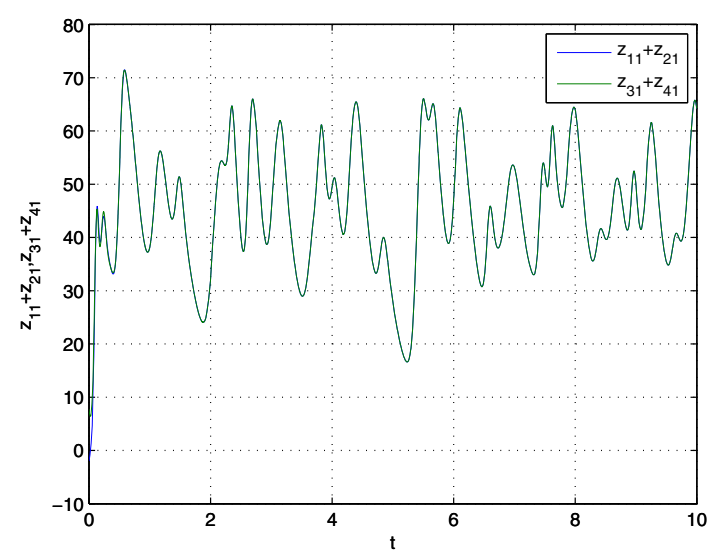

(c)

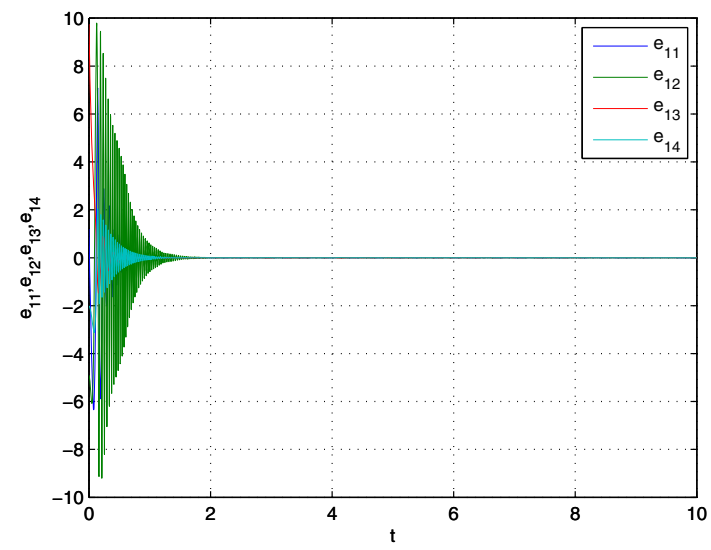

(e)

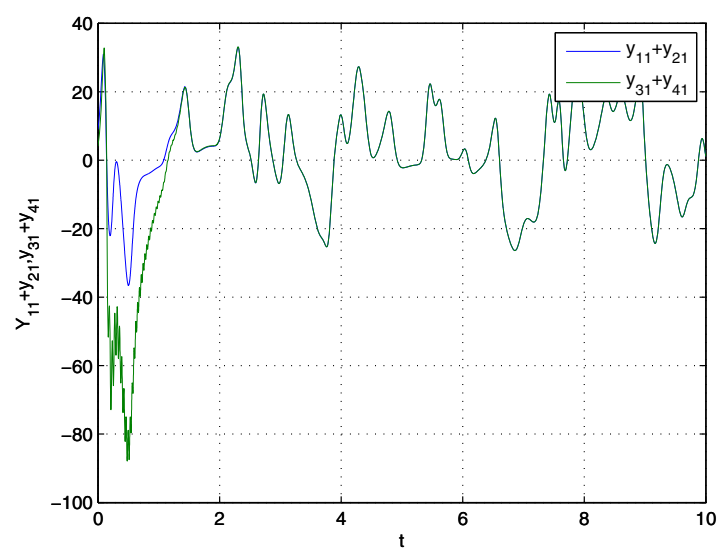

(b)

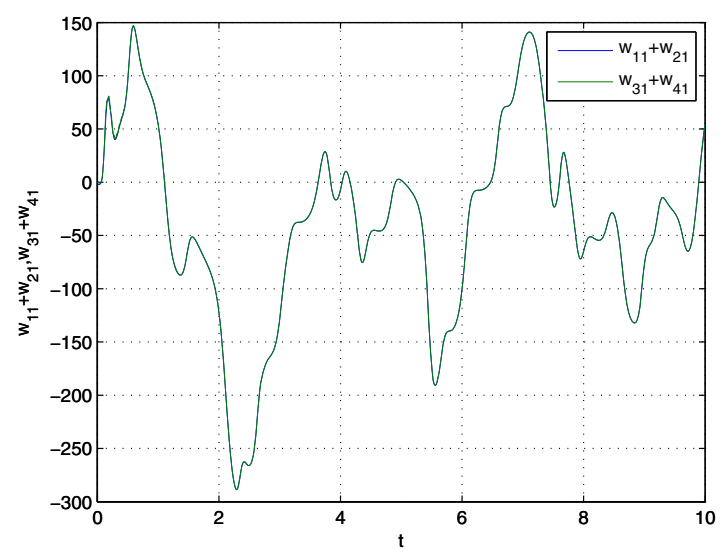

(d)



(f)

Fig. 3 Complete synchronization of hyperchaotic systems a between $x_{11}(t)+x_{21}(t)$ and $x_{31}(t)+x_{41}(t)$, b between $y_{11}(t)+y_{21}(t)$ and $y_{31}(t)+y_{41}(t)$, $\mathbf{c}$ between $z_{11}(t)+z_{21}(t)$ and $z_{31}(t)+z_{41}(t)$, $\mathbf{d}$ between $w_{11}(t)+w_{21}(t)$ and $w_{31}(t)+w_{41}(t)$, e synchronization errors $e_{11}, e_{12}, e_{13}, e_{14}, \mathbf{f}$ parameter estimation

the trajectories of master and slave variables with the control inputs. Also, initial states for error system are obtained as $\left(e_{11}, e_{12}, e_{13}, e_{14}\right)=(9.2,13.1,5.75,-2)$. The convergence of synchronization error $e(t)$ to zero as $t$ approaches infinity as exhibited in Fig. 4e establishes that the HPCCS among two master systems and two 


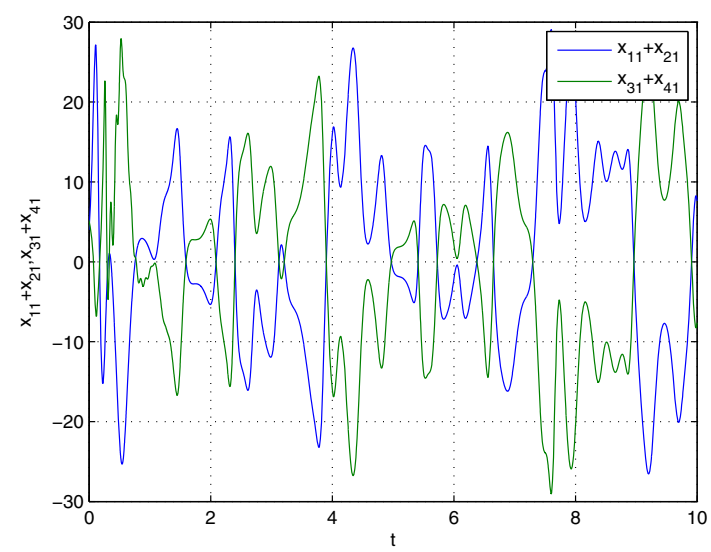

(a)

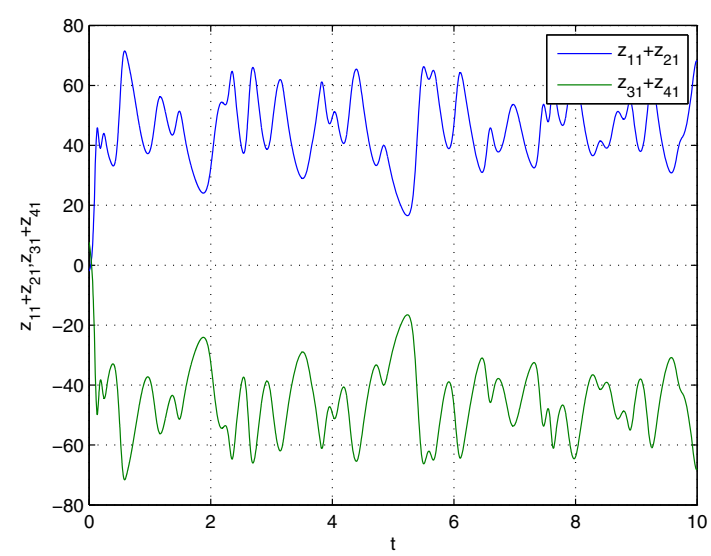

(c)

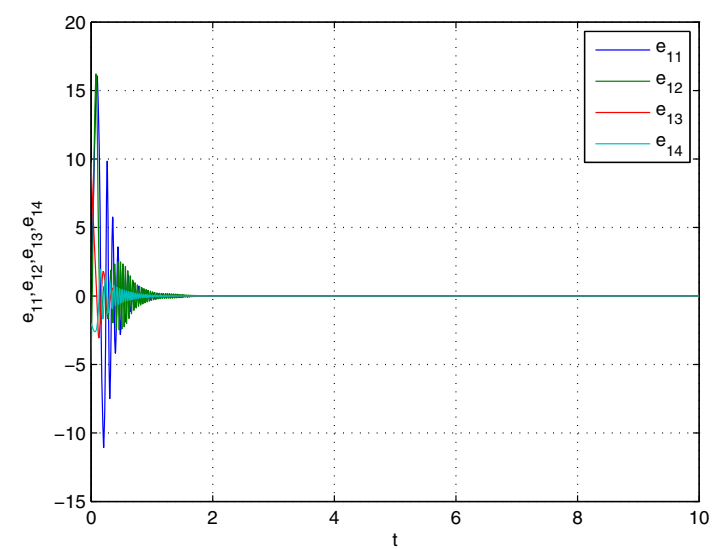

(e)

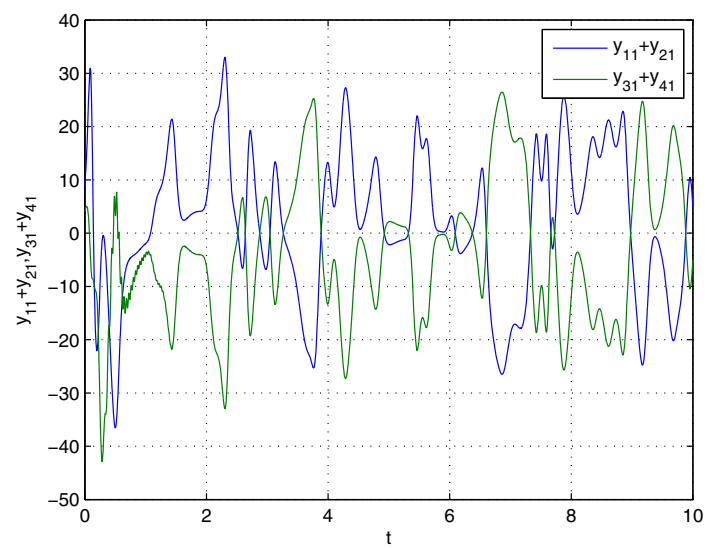

(b)

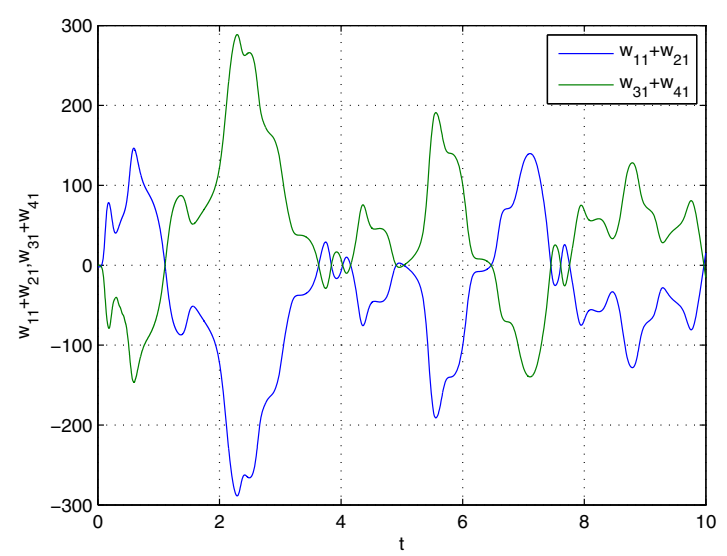

(d)

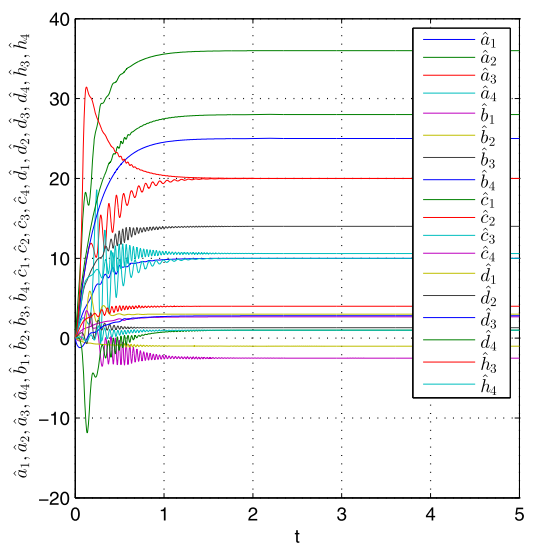

(f)

Fig. 4 Anti-synchronization of hyperchaotic systems a between $x_{11}(t)+x_{21}(t)$ and $x_{31}(t)+x_{41}(t)$, b between $y_{11}(t)+y_{21}(t)$ and $y_{31}(t)+y_{41}(t)$, c between $z_{11}(t)+z_{21}(t)$ and $z_{31}(t)+z_{41}(t)$, d between $w_{11}(t)+w_{21}(t)$ and $w_{31}(t)+w_{41}(t)$, e synchronization errors $e_{11}, e_{12}, e_{13}, e_{14}, \mathbf{f}$ parameter estimation

slave systems is achieved via ACM. Further, Fig. $4 \mathrm{f}$ displays that the estimated parameters converge to their respective original values asymptotically with time. Thus, the proposed HPCCS scheme between master and slave systems is justified computationally. 


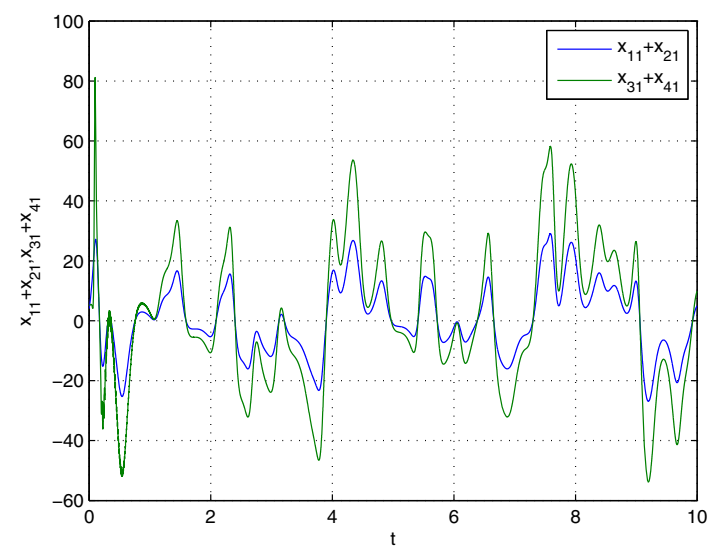

(a)

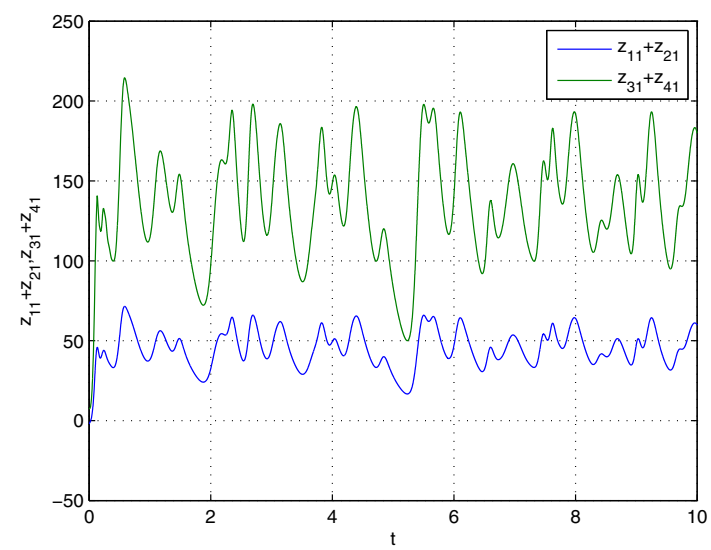

(c)

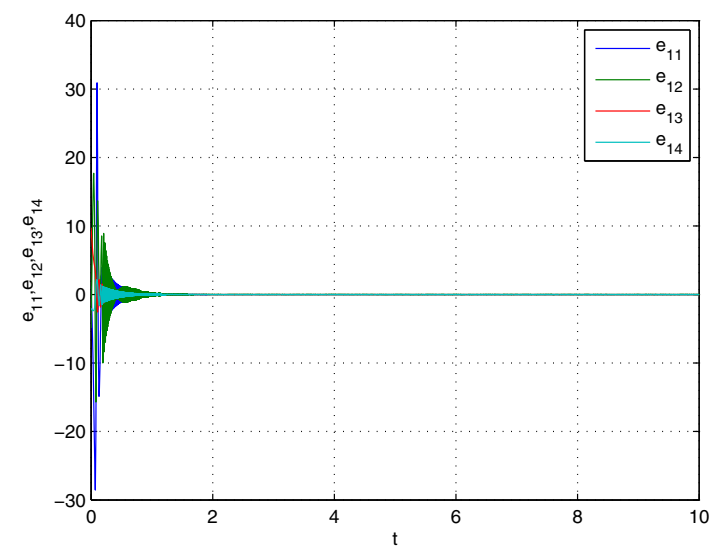

(e)

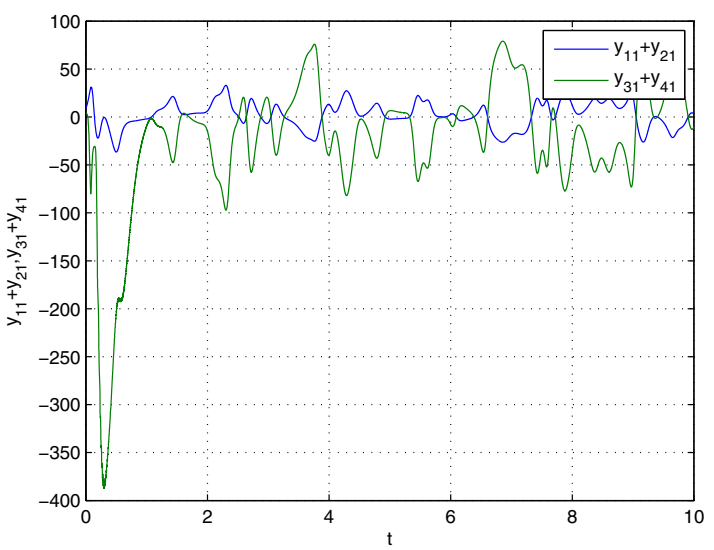

(b)

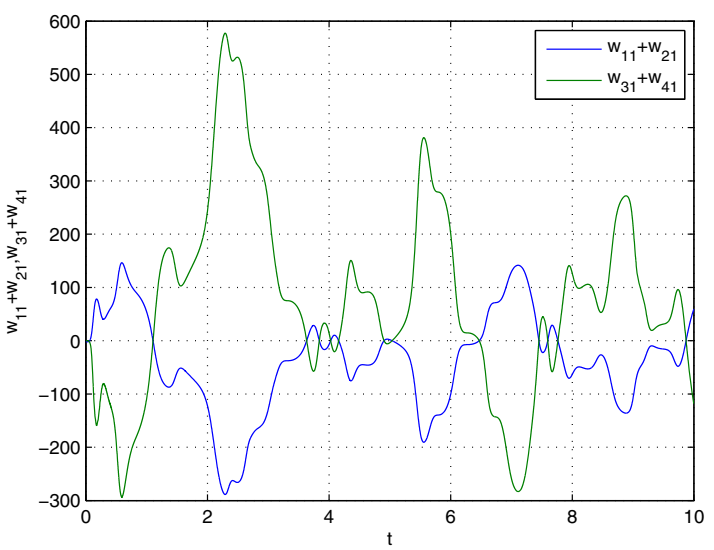

(d)



(f)

Fig. 5 HPCCS of hyperchaotic systems a between $x_{11}(t)+x_{21}(t)$ and $x_{31}(t)+x_{41}(t)$, b between $y_{11}(t)+y_{21}(t)$ and $y_{31}(t)+y_{41}(t)$, c between $z_{11}(t)+z_{21}(t)$ and $z_{31}(t)+z_{41}(t)$, d between $w_{11}(t)+w_{21}(t)$ and $w_{31}(t)+w_{41}(t)$, e HPCCS errors $e_{11}, e_{12}, e_{13}$, $e_{14}, \mathbf{f}$ parameter estimation

Case 3. In this case, we achieve HPCCS scheme of master and slave systems by choosing the scaling matrix $\alpha$ with $\alpha_{1}=2, \alpha_{2}=-3, \alpha_{3}=3, \alpha_{4}=-2$. Figure 5a-d displays the trajectories of master and slave variables with the control inputs. Further, initial states for error system are obtained as $\left(e_{11}, e_{12}, e_{13}, e_{14}\right)=$ 


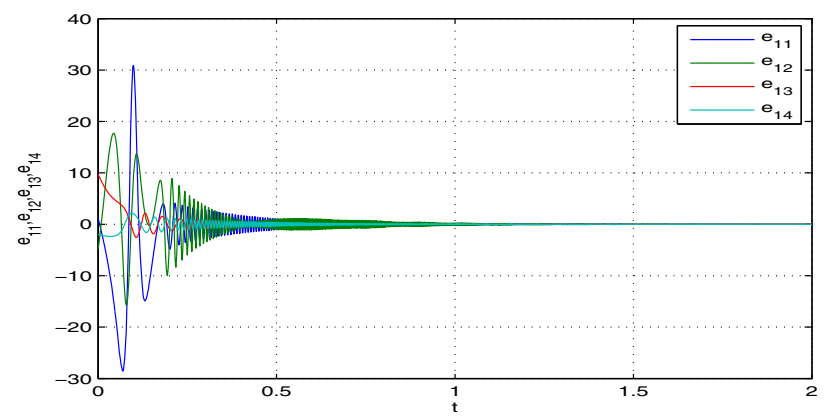

Fig. 6 HPCCS error convergence portrait

$(-2.8,31.1,13.75,-4)$. The convergence of synchronization error $e(t)$ to zero as $t$ approaches infinity as depicted in Fig. 5e ensures that the HPCCS among two master systems and two slave systems is achieved via ACM. Further, Fig. $5 \mathrm{f}$ exhibits that the estimated parameters converge to their respective original values asymptotically with time. Thus, the proposed HPCCS scheme between master and slave systems is ensured computationally.

A comparison analysis between the proposed HPCCS scheme and the earlier published work.

In Ref. [26], author used nonlinear active control to investigate the $\mathrm{C}-\mathrm{C}$ synchronization between four identical Lu systems and non-identical chaotic systems where it is noticed that the synchronization is obtained at $t=5$ (approx.) and $t=4.8$ (approx.), respectively. Also, author adopted sliding mode control approach in Ref. [41] to achieve $\mathrm{C}-\mathrm{C}$ synchronization among four different systems with uncertain parameters, here it has been found that synchronization error is converging to zero at $t=2.4$ (approx.). Further, in Ref. [42], author studied C-C synchronization among four complex nonlinear chaotic systems where it has been recorded that the error synchronization is realized at $t=5$ (approx.). Also in Ref. [45], author applied ACM to achieve $\mathrm{C}-\mathrm{C}$ synchronization between four identical $\mathrm{HC}$ systems where it noted that the synchronization state is attained at $t=5.1$ (approx.). Moreover, in Ref. [46], author utilized robust adaptive sliding mode control to generalize the $\mathrm{C}-\mathrm{C}$ synchronization of $n$-dimensional chaotic systems with time delay in which the synchronization is obtained at $t=4.9$ (approx.). Also in Ref. [48], author discussed phase synchronization between different complex chaotic systems of fractional order, by this procedure, the $\mathrm{C}-\mathrm{C}$ synchronization has been carried out at $t=4.5$ (approx.). Furthermore, in Ref. [55], author proposed ACM to study complex modified hybrid function projective synchronization between different complex chaotic systems with unknown complex parameters, where it has been seen that the desired synchronization is obtained at $t=4.5$ (approx.). Apart from the above-described studies, we have investigated the HPCCS scheme among four non-identical HC systems using ACM in which it has been found that the synchronization occurs at $t=1.2$ (approx.) as shown in Fig. 6. Hence, in comparison to the above discussed techniques, the synchronization time in our investigated scheme is much lesser which in turn shows the vitality and effectivity of the considered methodology.

\section{Conclusion}

In this paper, we have investigated the HPCCS scheme among four non-identical HC systems via ACM. The analytical expressions of control inputs and the parameter update laws are obtained in accordance with Lyapunov stability theory. Numerical simulations are demonstrated to check the effectivity and correctness of the theoretical results analysed by using MATLAB. Remarkably, the experimental outcomes and theoretical results are both in excellent compatibility. It is noticed that numerous synchronization schemes, viz., chaos control problem, combination synchronization, projective synchronization, etc. become the particular cases of $\mathrm{C}-\mathrm{C}$ synchronization. In our study, the synchronization error takes less time to converge to zero which implies that our proposed scheme is more preferred over earlier published work. The considered synchronization scheme may be used in secure communications and information processing with several applications in biological, social and physical systems.

Open Access This article is licensed under a Creative Commons Attribution 4.0 International License, which permits use, sharing, adaptation, distribution and reproduction in any medium or format, as long as you give appropriate credit to the original 
author(s) and the source, provide a link to the Creative Commons licence, and indicate if changes were made. The images or other third party material in this article are included in the article's Creative Commons licence, unless indicated otherwise in a credit line to the material. If material is not included in the article's Creative Commons licence and your intended use is not permitted by statutory regulation or exceeds the permitted use, you will need to obtain permission directly from the copyright holder. To view a copy of this licence, visit http://creativecommons.org/licenses/by/4.0/.

\section{References}

1. Li, Z.; Xu, D.: A secure communication scheme using projective chaos synchronization. Chaos Solitons Fractals 22(2), 477-481 (2004)

2. Provata, A.; Katsaloulis, P.; Verganelakis, D.A.: Dynamics of chaotic maps for modelling the multifractal spectrum of human brain diffusion tensor images. Chaos Solitons Fractals 45(2), 174-180 (2012)

3. Shi, Z.; Hong, S.; Chen, K.: Experimental study on tracking the state of analog Chua's circuit with particle filter for chaos synchronization. Phys. Lett. A 372(34), 5575-5580 (2008)

4. Tong, X.-J.; Zhang, M.; Wang, Z.; Liu, Y.; Ma, Jing: An image encryption scheme based on a new hyperchaotic finance system. Optik 126(20), 2445-2452 (2015)

5. Wang, X.; Vaidyanathan, S.; Volos, C.; Pham, V.-T.; Kapitaniak, Tomasz: Dynamics, circuit realization, control and synchronization of a hyperchaotic hyperjerk system with coexisting attractors. Nonlinear Dyn. 89(3), 1673-1687 (2017)

6. Russell, F.P.; Düben, P.D.; Niu, X.; Luk, W.; Palmer, Tim N: Exploiting the chaotic behaviour of atmospheric models with reconfigurable architectures. Comput. Phys. Commun. 221, 160-173 (2017)

7. Bouallegue, K.: A new class of neural networks and its applications. Neurocomputing 249, 28-47 (2017)

8. Ghosh, D.; Mukherjee, A.; Das, N.R.; Biswas, B.N.: Generation \& control of chaos in a single loop optoelectronic oscillator. Optik 165, 275-287 (2018)

9. Patle, B.K.; Parhi, D.R.K.; Jagadeesh, Anne; Kashyap, Sunil Kumar: Matrix-binary codes based genetic algorithm for path planning of mobile robot. Computers \& Electrical Engineering 67, 708-728 (2018)

10. Han, S.K.; Kurrer, C.; Kuramoto, Y.: Dephasing and bursting in coupled neural oscillators. Phys. Rev. Lett. 75(17), 3190 (1995)

11. Wu, G.-C.; Baleanu, D.; Lin, Z.-X.: Image encryption technique based on fractional chaotic time series. J. Vib. Control 22(8), 2092-2099 (2016)

12. Sahoo, B.; Poria, S.: The chaos and control of a food chain model supplying additional food to top-predator. Chaos Solitons Fractals 58, 52-64 (2014)

13. Poincaré, H.: Sur le problème des trois corps et les équations de la dynamique. Acta Mathematica 13(1), A3-A270 (1890)

14. Lorenz, E.N.: Deterministic nonperiodic flow. J. Atmos. Sci. 20(2), 130-141 (1963)

15. Fujisaka, H.; Yamada, T.: Stability theory of synchronized motion in coupled-oscillator systems. Prog. Theor. Phys. 69(1), 32-47 (1983)

16. Shinbrot, T.; Ott, E.; Grebogi, C.; Yorke, J.A.: Using chaos to direct trajectories to targets. Phys. Rev. Lett. 65(26), 3215 (1990)

17. Pecora, L.M.; Carroll, T.L.: Synchronization in chaotic systems. Phys. Rev. Lett. 64(8), 821 (1990)

18. Singh, A.K.; Yadav, V.K.; Das, S.: Synchronization between fractional order complex chaotic systems. Int. J. Dyn. Control 5(3), 756-770 (2017)

19. Li, C.; Liao, X.: Complete and lag synchronization of hyperchaotic systems using small impulses. Chaos Solitons Fractals 22(4), 857-867 (2004)

20. Li, G.-H.; Zhou, S.-P.: Anti-synchronization in different chaotic systems. Chaos Solitons Fractals 32(2), 516-520 (2007)

21. Sudheer, K.S.; Sabir, M.: Hybrid synchronization of hyperchaotic LU system. Pramana 73(4), 781 (2009)

22. Ding, Z:; Shen, Y.: Projective synchronization of nonidentical fractional-order neural networks based on sliding mode controller. Neural Netw. 76, 97-105 (2016)

23. Xu, Z.; Guo, L.; Hu, M.; Yang, Y.: Hybrid projective synchronization in a chaotic complex nonlinear system. Math. Comput. Simul. 79(1), 449-457 (2008)

24. Li, G.-H.: Modified projective synchronization of chaotic system. Chaos Solitons Fractals 32(5), 1786-1790 (2007)

25. Vincent, U.E.; Saseyi, A.O.; McClintock, P.V.E.: Multi-switching combination synchronization of chaotic systems. Nonlinear Dyn. 80(1-2), 845-854 (2015)

26. Sun, J.; Shen, Y.; Zhang, G.; Chengjie, X.; Cui, Guangzhao: Combination-combination synchronization among four identical or different chaotic systems. Nonlinear Dyn. 73(3), 1211-1222 (2013)

27. Sun, J.; Shen, Y.; Yin, Q.; Xu, C.: Compound synchronization of four memristor chaotic oscillator systems and secure communication. Chaos Interdiscip. J. Nonlinear Sci. 23(1), 013140 (2013)

28. Delavari, H.; Mohadeszadeh, M.: Hybrid complex projective synchronization of complex chaotic systems using active control technique with nonlinearity in the control input. J. Control Eng. Appl. Inf. 20(1), 67-74 (2018)

29. Vaidyanathan, S.; Sampath, S.: Anti-synchronization of four-wing chaotic systems via sliding mode control. Int. J. Autom. Comput. 9(3), 274-279 (2012)

30. Khan, A.; Bhat, M.A.: Hyper-chaotic analysis and adaptive multi-switching synchronization of a novel asymmetric non-linear dynamical system. Int. J. Dyn. Control 5(4), 1211-1221 (2017)

31. Khan, A.; Tyagi, A.: Analysis and hyper-chaos control of a new 4-D hyper-chaotic system by using optimal and adaptive control design. Int. J. Dyn. Control 5(4), 1147-1155 (2017)

32. Rasappan, S.; Vaidyanathan, S.: Synchronization of hyperchaotic Liu system via backstepping control with recursive feedback. In: Mathew J., Patra P., Pradhan D.K., Kuttyamma A.J. (eds.) Eco-friendly Computing and Communication Systems. ICECCS 2012. Communications in Computer and Information Science, vol 305, pp. 212-221. Springer, Berlin, Heidelberg (2012) 
33. Li, D.; Zhang, X.: Impulsive synchronization of fractional order chaotic systems with time-delay. Neurocomputing 216, 39-44 (2016)

34. Liao, X.; Chen, G.: Chaos synchronization of general Lur'e systems via time-delay feedback control. Int. J. Bifurc. Chaos 13(01), 207-213 (2003)

35. Rossler, O.E.: An equation for hyperchaos. Phys. Lett. A 71(2-3), 155-157 (1979)

36. Hubler, A.W.: Adaptive control of chaotic system. Helv. Phys. Acta 62, 343-346 (1989)

37. Liao, T.-L.; Tsai, S.-H.: Adaptive synchronization of chaotic systems and its application to secure communications. Chaos Solitons Fractals 11(9), 1387-1396 (2000)

38. Yassen, M.T.: Adaptive control and synchronization of a modified Chua's circuit system. Appl. Math. Comput. 135(1), $113-128(2003)$

39. Mainieri, R.; Rehacek, J.: Projective synchronization in three-dimensional chaotic systems. Phys. Rev. Lett. 82(15), 3042 (1999)

40. Wu, Z.; Duan, J.; Fu, X.: Complex projective synchronization in coupled chaotic complex dynamical systems. Nonlinear Dyn. 69(3), 771-779 (2012)

41. Sun, J.; Shen, Y.; Wang, X.; Chen, J.: Finite-time combination-combination synchronization of four different chaotic systems with unknown parameters via sliding mode control. Nonlinear Dyn. 76(1), 383-397 (2014)

42. Zhou, X., Xiong, L., Cai, X.: Combination-combination synchronization of four nonlinear complex chaotic systems. In: Abstract and Applied Analysis, vol. 2014. Hindawi (2014)

43. Mahmoud, G.M.; Abed-Elhameed, T.M.; Ahmed, M.E.: Generalization of combination-combination synchronization of chaotic $n$-dimensional fractional-order dynamical systems. Nonlinear Dyn. 83(4), 1885-1893 (2016)

44. Sun, J.; Wang, Y.; Cui, G.; Shen, Y.: Dynamical properties and combination-combination complex synchronization of four novel chaotic complex systems. Optik Int. J. Light Electron Opt. 127(4), 1572-1580 (2016)

45. Khan, A.; et al.: Chaotic analysis and combination-combination synchronization of a novel hyperchaotic system without any equilibria. Chin. J. Phys. 56(1), 238-251 (2018)

46. Khan, Ayub; Singh, Shikha: Generalization of combination-combination synchronization of $n$-dimensional time-delay chaotic system via robust adaptive sliding mode control. Math. Methods Appl. Sci. 41(9), 3356-3369 (2018)

47. Khan, A.; Singh, S.; Azar, A.T.: Combination-combination anti-synchronization of four fractional order identical hyperchaotic systems. In: International Conference on Advanced Machine Learning Technologies and Applications, pp. 406-414. Springer (2019)

48. Yadav, V.K.; Prasad, G.; Srivastava, M.; Das, S.: Combination-combination phase synchronization among non-identical fractional order complex chaotic systems via nonlinear control. Int. J. Dyn. Control 7(2), 1-11 (2018)

49. Runzi, L.; Yinglan, W.: Finite-time stochastic combination synchronization of three different chaotic systems and its application in secure communication. Chaos Interdiscip. J. Nonlinear Sci. 22(2), 023109 (2012)

50. Wu, Z.; Fu, X.: Combination synchronization of three different order nonlinear systems using active backstepping design. Nonlinear Dyn. 73(3), 1863-1872 (2013)

51. Wang, Xingyuan; Wang, Mingjun: A hyperchaos generated from lorenz system. Physica A Stat. Mech. Appl. 387(14), $3751-3758(2008)$

52. Chen, A.; Junan, L.; Lü, J.; Simin, Y.: Generating hyperchaotic lü attractor via state feedback control. Physica A Stat. Mech. Appl. 364, 103-110 (2006)

53. Zheng, S.; Dong, G.; Bi, Q.: A new hyperchaotic system and its synchronization. Appl. Math. Comput. 215(9), 3192-3200 (2010)

54. Wei, Z.; Wang, R.; Liu, A.: A new finding of the existence of hidden hyperchaotic attractors with no equilibria. Math. Comput. Simul. 100, 13-23 (2014)

55. Liu, J.; Liu, S.; Sprott, JCl: Adaptive complex modified hybrid function projective synchronization of different dimensional complex chaos with uncertain complex parameters. Nonlinear Dyn. 83(1-2), 1109-1121 (2016)

Publisher's Note Springer Nature remains neutral with regard to jurisdictional claims in published maps and institutional affiliations. 